\title{
High-Power Coupler Component Test Stand Status and Results
}

B. Rusnak, F. Wang, C. Adolphsen, G. Bowden, C. Nantista, R. Swent, J. Tice, B. McKee, K. Jobe, Z. Li, L. $\mathrm{Ge}$

June 20, 2007

2007 Particle Accelerator Conference Albuquerque, NM, United States June 25, 2007 through June 29, 2007 
This document was prepared as an account of work sponsored by an agency of the United States Government. Neither the United States Government nor the University of California nor any of their employees, makes any warranty, express or implied, or assumes any legal liability or responsibility for the accuracy, completeness, or usefulness of any information, apparatus, product, or process disclosed, or represents that its use would not infringe privately owned rights. Reference herein to any specific commercial product, process, or service by trade name, trademark, manufacturer, or otherwise, does not necessarily constitute or imply its endorsement, recommendation, or favoring by the United States Government or the University of California. The views and opinions of authors expressed herein do not necessarily state or reflect those of the United States Government or the University of California, and shall not be used for advertising or product endorsement purposes. 


\title{
HIGH-POWER COUPLER COMPONENT TEST STAND STATUS AND RESULTS*\#
}

\author{
B. Rusnak, LLNL, Livermore, CA 94550 \\ F. Wang, C. Adolphsen, G. Bowden, C. Nantista, R. Swent, J. Tice, B. McKee, \\ K. Jobe, Z. Li, L. Ge, SLAC, Menlo Park, CA 94025
}

\begin{abstract}
Fundamental power couplers for superconducting accelerator applications like the ILC are complicated transmission line assemblies that must simultaneously accommodate demanding RF power, cryogenic, and cleanliness constraints. When these couplers are RF conditioned, the observed response is an aggregate of all the parts of the coupler and the specific features that dominate the conditioning response are hard to determine. To better understand and characterize RF conditioning phenomena toward improving performance and reducing conditioning time, a high-power coupler component test stand has been built at SLAC. Operating at $1.3 \mathrm{GHz}$, this test stand was designed to measure the conditioning behavior of select components of the TTFIII coupler independently, including outer-conductor bellows, tube transitions, copper plating, surface preparations, and cold window geometries and coatings. A description of the test stand, the measurement approach, and a summary of the results obtained so far are presented.
\end{abstract}

\section{INTRODUCTION}

To understand the long processing time observed in TTFIII power couplers, a coupler component test stand has been operating at SLAC and a variety of coupler parts are being tested. RF power, vacuum pressure, and electron probe signals are being measured to better understand which components and preparation processes most impact conditioning. The RF conditioning procedure starts with short, $20 \mu \mathrm{s}$, RF pulses. As long as the measured ion pump current levels are lower than the threshold of $50 \mu \mathrm{A}$ $\left(\sim 2.5 \times 10^{-7}\right.$ Torr $)$, the power is increased stepwise to a maximum level. From here, the maximum power is then reduced to a minimum value and then ramped up as before but with longer pulse widths, as illustrated in Figure 3. Any increase of pressure above the threshold results in a power reduction. This approach is similar to that done at other labs [1,2]. An electron signal is measured from a pickup probe on the device under test and is useful for diagnosing multipacting and electron activity.

\section{TEST STAND SET UP}

In order to test specific features of TTFIII power coupler as to their impact on RF conditioning and ultimate performance, the test stand shown in Figure 1 was built.

\footnotetext{
*This work was performed under the auspices of the U.S. Department of Energy by the University of California, Lawrence Livermore National Laboratory under Contract No. W-7405-Eng-48.
}

${ }^{*}$ Work Supported by DOE Contract DE-AC02-76F00515.
High power RF is delivered from pressurized WR650 waveguide through a vacuum window to a customdesigned waveguide-to-coaxial transition. After the transition, sections of coaxial transmission line, the Device(s) Under Test (DUT), are installed. The RF is then routed to a load through another waveguide-to-coaxial transition, window and waveguide. Powered by a recently-installed $5 \mathrm{MW}, 1.3 \mathrm{GHz}$ klystron at SLAC, the test stand allows for operation at RF levels higher than required for the ILC.

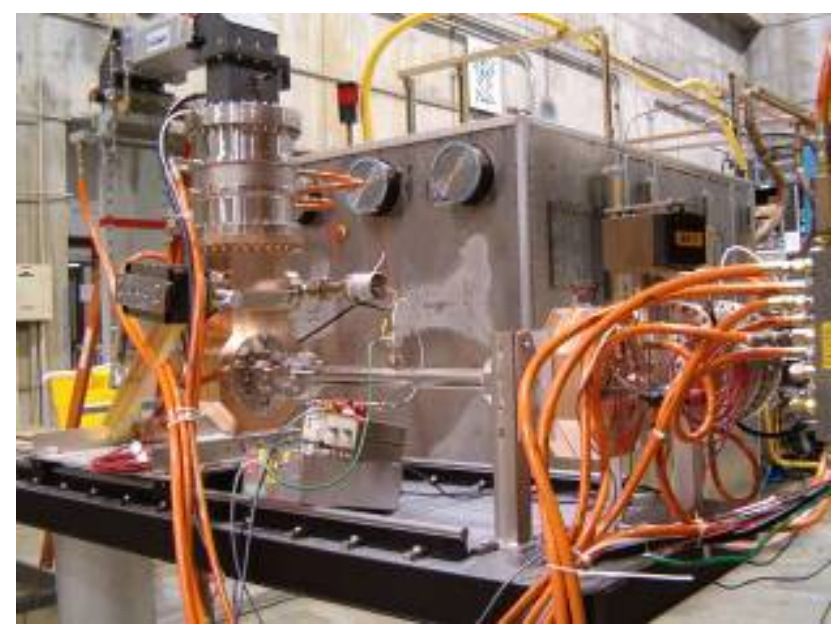

Figure 1. Photo of the SLAC high-power coupler component test stand. Shown are the round ceramic vacuum windows, the copper WR650 waveguide-tocoaxial transitions and a $610 \mathrm{~mm}$ long, $40 \mathrm{~mm}$ outer diameter, stainless steel, coaxial DUT between them.

The objective of the test stand is to accurately measure the vacuum and electron response of the system while powered. To avoid arcing, special attention was paid to ensure the electrical connection of the center conductor was robust and reproducible. To avoid increased outgassing from RF heating, the materials and geometry were chosen to limit the temperature rise of the inner conductor. Still, thermal expansion needed to be considered when the inner and outer conductors are made of different materials.

Accommodating these constraints was accomplished by having a solid copper inner conductor that was screw mounted to one water-cooled base on the waveguide-tocoaxial transition, while the other side was firmly held by a four-jaw beryllium-copper sliding joint assembly that was mounted to the water-cooled base on the other waveguide-to-coaxial transition. The design detail of the 


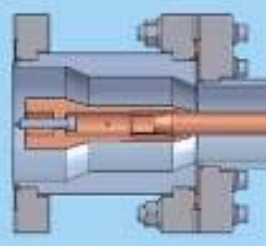

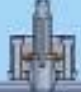

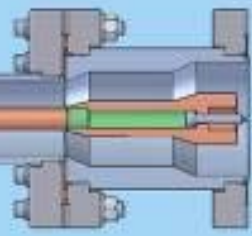

Figure 2. Drawing of the step transitions and DUT (straight $40 \mathrm{~mm}$ diameter tube) showing the inner conductor screw-anchored on the right side (green area) and the sliding Be-Cu connection on the left side.

inner conductor configuration and connectors is shown in Figure 2.

\section{TEST PLAN}

In order to evaluate which features of the TTFIII design most affect the conditioning performance of the overall coupler, each feature will be measured separately as is feasible. By separately testing individual or subsets of components, we hope to identify which aspects of this coupler design are problematic.

The first stage of testing is being done on a straight 70 Ohm coax with a $40 \mathrm{~mm}$ diameter outer conductor to look at the effects of surface coatings and a variety of preparations. This test emulates the cold part of the TTFIII coupler design. Initial tests have been done with a honed stainless steel outer conductor: future tests will be of copper-plated straight outer conductors that are made using the CPI/DESY plating recipe.

The second stage of testing will be with a bellows section welded into the outer conductor. The whole section will be copper plated. This test should provide data on the effects of bellows on conditioning times, which are important for SRF couplers as bellows not only provide mechanical compliance in cryomodule assemblies, but also are high-quality thermal breaks that allow a coupler to be shorter.

The third stage of testing will investigate the impact of the cold window assembly on conditioning. This will be done in two parts. The first part will test the transmission line in the area of the window with the ceramic installed. The second part will test the area with a modification that allows well-matched operation without the ceramic present. Thus, the impact on conditioning from the ceramic itself can be better evaluated.

\section{INITIAL RESULTS}

Initial testing of a straight, stainless steel wall, $40 \mathrm{~mm}$ diameter coaxial tube has provided operating experience and a plethora of vacuum pressure and electron probe data that are being evaluated to gain insights for latter tests. The pressure response from repeated ramping of the RF power is shown in Figure 3. After the initial ramp cycle at short pulse, the multipacting bands become more apparent and are in fair agreement with that expected from simulations. Figure 4 is an example of the pressure and electron signal response from repeated power ramps, and Figure 5 shows the expected multipacting bands by their order.

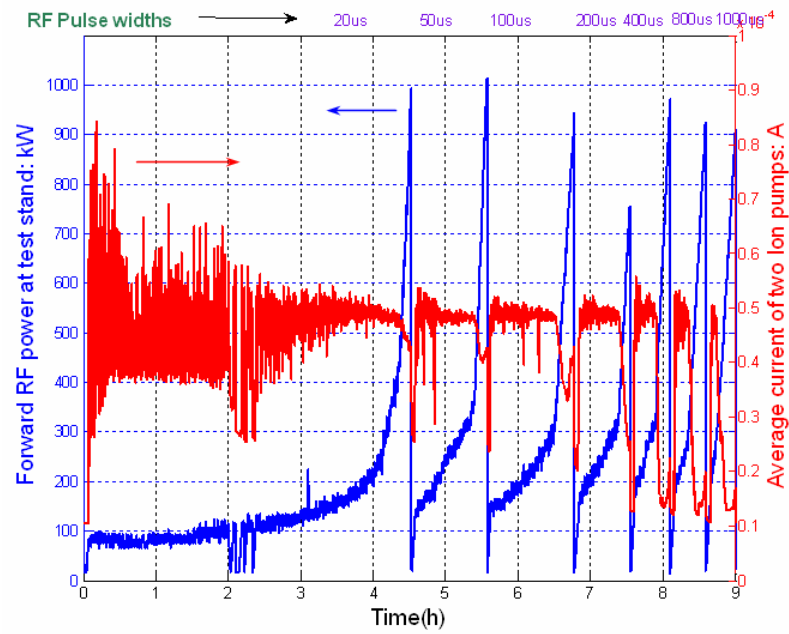

Figure 3. Initial processing results of a stainless steel coaxial tube showing RF power (blue) and vacuum response (red).

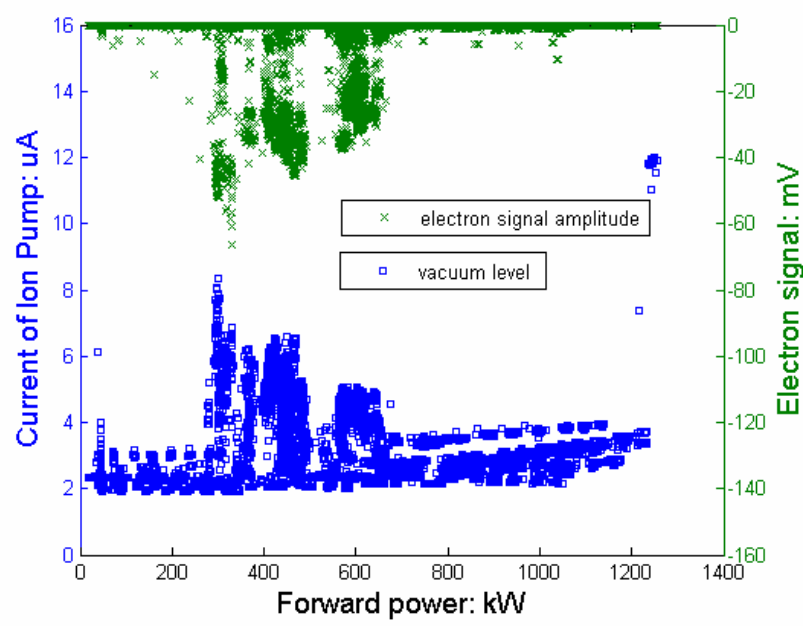

Figure 4. Plot of vacuum and electron signal activity during repeated RF power ramps.

Electron activity during conditioning was measured by an electron pickup probe, and had a number of interesting characteristics. One was that after the RF pulse reached 


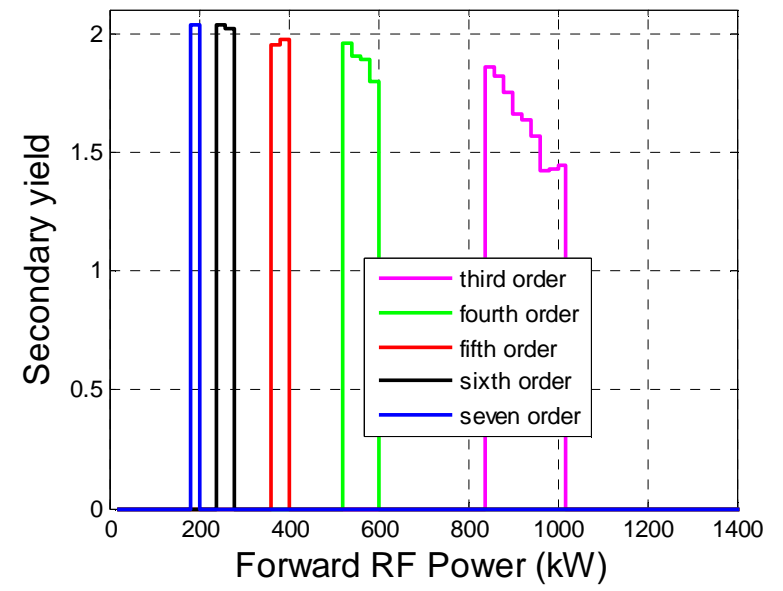

Figure 5. Plot showing the simulated multipacting bands by order (i.e., how may RF cycles before impact).

full power, the turn on the electron signal was delayed from a few microseconds to over ten microseconds depending on whether the vacuum pressure was high or low. When it did turn on, it was very rapid with typical transition times of $50 \mathrm{~ns}$. A spectra analysis of the signal showed it to be rich in higher harmonics of the $1.3 \mathrm{GHz}$ RF frequency. Also, the electron signal was present even with no bias on the probe, and when a voltage bias was applied, the amplitude of the detected signal changed significantly, as shown in Figure 6. The shape and temporal character of the probe signal was highly dependent on the RF power level as was the delay time. Placing a small magnet anywhere along the $610 \mathrm{~mm}$ length of the coaxial section would cause the delay of the electron signal to go to zero immediately. Immersing a 20 $\mathrm{cm}$ section of the $610 \mathrm{~mm}$ coax in a solenoidal field up to

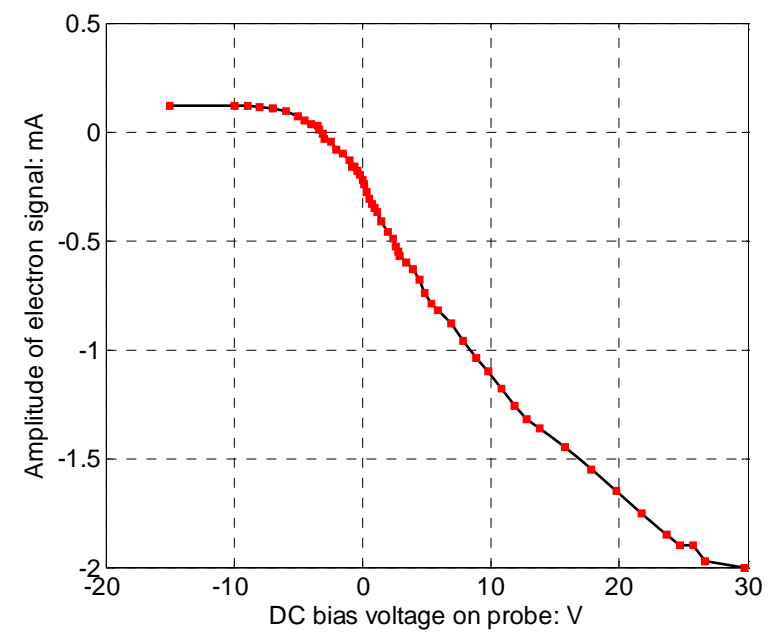

Figure 6. The amplitude of electron signal at different DC bias voltages with $620 \mathrm{~kW}$ of RF input power and a vacuum level of 2e-7 Torr. At larger negative biases (up to a $1 \mathrm{kV}$ ), the signal slowly reduces to zero, suggesting that the positive signal in this regime is from secondary emission off the probe.
300 Gauss also eliminated the delay time but broadened the multipacting response. The 300 Gauss level is expected to suppress multipacting, but the lower fields on either side of the solenoid widened the response there.

\section{DISCUSSION}

Although the experimental results and simulations of multipacting are in fair agreement in scale, the latter show distinct multipacting bands while the former are broader and less clearly delineated. This may be attributed to the thermal velocity spread of emitted secondary electrons and the random delay time of secondary electron emission relative to the RF wave, which are not included in the simulations. As discussed in [3], electron thermal distributions and emission onset delay can affect the multipacting phase width, and hence could shift and broaden the multipacting power levels. The differences may also be related to the fact that electron space charge effects are not included in the simulations.

Regarding the observed delay in the electron signal after the start of the RF pulse, it has been suggested that this is due to there being insufficient electrons available to seed the onset of multipacting [4]. To see if such electrons could be provided, a short (1 microsecond), high power (3 MW) RF spike was included at beginning of the pulse. After this spike, the electron signal turned on immediately, suggesting that field emission electrons were seeding the start of multipacting in this case.

\section{CONCLUSION}

The coupler component test stand at SLAC works well and is capable of producing component-specific data and insights into what features and aspects of a coupler design most affects its conditioning performance. We are in the process of testing other parts and subassemblies of the TTFIII coupler, which we hope will lead to a better understanding the processing limitations and to methods of improving the long-term performance of couplers for the ILC SRF cavities.

\section{REFERENCES}

[1] B. Dwersteg et al., "TESLA RF Power Couplers Development at DESY", Proceedings of the SRF2001 Workshop, Tsukuba (Japan).

[2] H. Jenhani et al., "Developments in Conditioning Procedures for the TTF-III Power Couplers", Proceedings of EPAC 2006, Edinburgh (Scotland).

[3] Spilios Riyopoulos et al., "Effect of Random Secondary Delay Times and Emission Velocities in Electron Multipactors", IEEE Transactions on Electron Devices, Vol. 44, No.3, Mar. 1997.

[4] D. Raboso, A. Woode, "A New Method of Electron Seeding Used for Accurate Testing of Multipactor Transients", Vol.1, Oct. 1995. 